Research Article

\title{
Comparison of multiple genotyping methods for the identification of the cancer predisposing founder mutation p.R337H in TP53.
}

\author{
Mariana Fitarelli-Kiehl ${ }^{1,2}$, Gabriel S. Macedo ${ }^{1,2}$, Rosane Paixão Schlatter ${ }^{3}$, Patricia Koehler-Santos ${ }^{4}$, Ursula \\ da Silveira Matte ${ }^{1,4,5}$, Patricia Ashton-Prolla ${ }^{1,2,5}$, Juliana Giacomazzi ${ }^{2,5}$ \\ ${ }^{1}$ Programa de Pós-Graduação em Genética e Biologia Molecular, \\ Universidade Federal do Rio Grande do Sul (UFRGS), Porto Alegre, RS, Brazil. \\ ${ }^{2}$ Laboratório de Medicina Genômica, Centro de Pesquisa Experimental, \\ Hospital de Clínicas de Porto Alegre (HCPA), Porto Alegre, RS, Brazil. \\ ${ }^{3}$ Grupo de Pesquisa e Pós-Graduação (GPPG), Hospital de Clínicas de Porto Alegre and Programa de Pós \\ Graduação em Cardiologia, UFRGS Porto Alegre, RS, Brazil. \\ ${ }^{4}$ Unidade de Análises Moleculares e de Proteinas (UAMP), Centro de Pesquisa Experimental, \\ Hospital de Clínicas de Porto Alegre, Porto Alegre, RS, Brazil. \\ ${ }^{5}$ Departamento de Genética, Universidade Federal do Rio Grande do Sul (UFRGS), \\ Porto Alegre, RS, Brazil.
}

\begin{abstract}
Germline mutations in the TP53 gene are associated with Li-Fraumeni and Li-Fraumeni-Like Syndromes, characterized by increased predisposition to early-onset cancers. In Brazil, the prevalence of the TP53-p.R337H germline mutation is exceedingly high in the general population and in cancer-affected patients, probably as result of a founder effect. Several genotyping methods are used for the molecular diagnosis of LFS/LFL, however Sanger sequencing is still considered the gold standard. We compared performance, cost and turnaround time of Sanger sequencing, PCR-RFLP, TaqMan-PCR and HRM in the p.R337H genotyping. The performance was determined by analysis of 95 genomic DNA samples and results were $100 \%$ concordant for all methods. Sequencing was the most expensive method followed by TaqMan-PCR, PCR-RFLP and HRM. The overall cost of HRM increased with the prevalence of positive samples, since confirmatory sequencing must be performed when a sample shows an abnormal melting profile, but remained lower than all other methods when the mutation prevalence was less than $2.5 \%$. Sequencing had the highest throughput and the longest turnaround time, while TaqMan-PCR showed the lowest turnaround and hands-on times. All methodologies studied are suitable for the detection of $\mathrm{p} . \mathrm{R} 337 \mathrm{H}$ and the choice will depend on the application and clinical scenario.
\end{abstract}

Keywords: TP53-p.R337H, RFLP, TaqMan, HRM, Sanger Sequencing.

Received: November 30, 2014; Accepted: September 22, 2015.

\section{Introduction}

Li-Fraumeni and Li-Fraumeni-Like Syndromes (LFS/LFL; OMIM\# 151623) are autosomal dominant disorders characterized by increased predisposition to multiple early-onset cancers caused by germline mutations in the TP53 gene (Malkin et al., 1990). In Europe and North America, germline TP53 mutations occur in approximately 1 in 5,000 live births (Lalloo et al., 2006; Gonzalez et al., 2009). In Brazil, a specific germline TP53 mutation, p.R337H (c.1010G > A; exon 10, also known as

Send correspondence to Patricia Ashton-Prolla. Laboratório de Medicina Genômica, Centro de Pesquisa Experimental, Hospital de Clínicas de Porto Alegre, Rua Ramiro Barcelos, 2350, 90035903 Porto Alegre, RS, Brazil. E-mail: pprolla@ @cpa.edu.br.
p.Arg337His), has been described at high frequency not only in the general population of southern Brazil but also in different cohorts of patients with cancer. Carrier frequencies of 1:300 have been reported in the Brazilian States of Paraná (newborn screening program) and Rio Grande do Sul (women enrolled in a breast cancer screening cohort) (Palmero et al., 2008; Custodio et al., 2013). Among breast cancer-affected women unselected by family history of cancer, p.R337H has been described at a frequency of up to $8.6 \%$, and reached $12.1 \%$ in women diagnosed with breast cancer at or before age 45 (Giacomazzi et al., 2013; Cury et al., 2014; Giacomazzi et al., 2014). In children with adrenocortical or choroid plexus carcinomas, the same alteration has been reported at a frequency of $90 \%$ (Ribeiro et al., 2001; Achatz et al., 2007; Seidinger et al., 2011). Thus, 
the prevalence of this mutation in the general population and in cancer-affected patients in Brazil is exceedingly high, probably due to a founder effect (Garritano et al., 2010), classifying it as the most common germline TP53 mutation ever described in any population. In addition to compulsory testing for the mutation in the State of Paraná since 2005, some investigators have suggested that any woman diagnosed with premenopausal breast cancer (especially when associated with a positive family history of breast cancer) in southern Brazil should be screened for p.R337H (Garritano et al., 2010; Euhus and Robinson, 2013; Giacomazzi et al., 2014).

Several genotyping methods have been proposed and are routinely used in clinical practice for the molecular diagnosis of LFS and LFL. Gene sequencing, however, is still considered the gold standard diagnostic method for identification of germline mutations in genes with high allelic heterogeneity, such as TP53. However, it is still relatively expensive and laborious and requires extensive automation, instrumentation and data interpretation. Thus, to interrogate the presence of a single mutation, alternative and less expensive site-specific testing strategies could be used. Among these, PCR-RFLP (Polymerase Chain Reaction followed by Restriction Fragment Length Polymorphism analysis), a traditional genotyping method, requires that the sequence variation under study generates or abolishes a restriction enzyme recognition site (Narayanan, 1991). After PCR amplification, the resulting DNA fragment is digested by one or more specific endonucleases that recognize restriction sites, resulting in fragments of different sizes that are then resolved by gel electrophoresis. Although this technique does not require sophisticated instruments, it is laborious and fully manual, which limits the number of analyses that can be performed in each experiment.

Allelic discrimination using TaqMan-PCR, is another mutation-specific diagnostic method which combines real-time PCR amplification and detection into a single step. Each TaqMan genotyping assay consists of two allele-specific TaqMan minor groove binding (MGB) probes containing distinct fluorescent dyes and a PCR primer pair for amplifying the sequence of interest. Cleavage of the fluorogenic probes during amplification liberates reporter dyes and its fluorescent signals indicate the allele(s) present in each sample (Livak 1999). Finally, high-resolution DNA melting analysis (HRM) was introduced in the early 2000's as a simple and inexpensive method for genotyping and mutation scanning (Wittwer et al., 2003). HRM is a closed-tube mutation screening method that requires no post-PCR processing of the samples and uses specific saturation dyes that fluoresce only in the presence of double stranded DNA. After real-time PCR amplification, the fragment's melting pattern is generated by monitoring the fluorescence over a temperature range. Homozygous, heterozygous and wild type samples are distinguished according to their melting profile and melting temperatures $\left(\mathrm{T}_{\mathrm{m}}\right)$.
In contrast with PCR-RFLP and TaqMan assays, however, high resolution melting (HRM) is a screening method which interrogates mutations in a given PCR-amplified DNA region, but it does not allow, in most cases, precise identification of the mutation. Hence, a second, confirmation step such as DNA sequencing is required for definitive mutation diagnosis (Reed et al., 2007).

In this study, we compare the performance and cost of these four different diagnostic approaches in the identification of the founder Brazilian mutation TP53-p.R337H.

\section{Material and Methods}

\section{Subjects}

DNA samples from 95 p.R337H carriers and noncarriers identified in previous research studies from our laboratory (IRB protocols 08-022 and 08-080, GPPG/HCPA), were included in this study. All individuals had consented to TP53 genotyping for diagnostic purposes and signed an informed consent.

\section{DNA isolation and quantification}

Genomic DNA was isolated from $200 \mu \mathrm{L}$ whole blood using the lllustra ${ }^{\mathrm{TM}}$ Blood GenomicPrep Mini Spin Kit (GE Healthcare, UK), according to the manufacturer's instructions. DNA concentration and purity were determined using a NanoDrop 1000 spectrophotometer (Thermo Scientific, USA).

\section{TP53-p.R337H genotyping}

\section{Sanger DNA Sequencing}

TP53 exon 10 sequencing involves PCR amplification of exon 10, purification of PCR fragments, cycle sequencing and purification of sequencing products. PCR was performed using primers and conditions previously described (primer sequences and PCR conditions are available at http://p53.iarc.fr/Download/ TP53_DirectSequencing_IARC.pdf; Petitjean et al., 2007) and then treated with $10 \mathrm{U}$ of Exonuclease I and $0.5 \mathrm{U}$ of Shrimp Alkaline Phosphatase (Fermentas), and incubated at $37^{\circ} \mathrm{C}$ for $30 \mathrm{~min}$ and at $80^{\circ} \mathrm{C}$ for $15 \mathrm{~min}$. Cycle sequencing was performed using BigDye Terminator kit version 3.1 (Applied Biosystems, USA) and the extension products were purified with BigDye XTerminator Purification Kit (Applied Biosystems, USA) according to the manufacturer's instructions. Sequencing products were analyzed on a 3500 Genetic Analyzer (Applied Biosystems, USA). Sequencing data visualization and sequence alignment were done with Chromas v2.0 and CLC Main Workbench (CLC Bio, DK) softwares, respectively.

\section{PCR-RFLP assay}

PCR (TP53 exon 10) was performed according to previously published protocols (Petitjean et al., 2007). PCR products were cleaved with $\mathrm{Hha \textrm {I }}$ at $37^{\circ} \mathrm{C}$ for $2 \mathrm{~h}$ and then 
resolved in 3\% agarose gels stained with GelRed ${ }^{\mathrm{TM}}$ (Biotium, USA). Resulting fragments were: 238 bp (homozygous mutant, AA genotype), $238 \mathrm{bp}, 146 \mathrm{bp}$ and $92 \mathrm{bp}$ (heterozygote, GA genotype) and $146 \mathrm{bp}$ and $92 \mathrm{bp}$ (homozygous wild-type, GG genotype).

\section{TaqMan-PCR}

Custom made allele-specific TaqMan ${ }^{\circledR}$ probes were used (Applied Biosystems, USA; Assay ID TP53R337H; AHBJWZJ). Real-time PCR reactions were done in a final volume of $12.5 \mu \mathrm{L}$, containing $20 \eta$ g of genomic DNA, $1 \mathrm{X}$ TaqMan Universal PCR Master Mix and 1X Custom TaqMan R337H Genotyping Assay. Cycling conditions were as follows: initial denaturation at $95^{\circ} \mathrm{C}$ for $10 \mathrm{~min}, 40$ cycles at $92^{\circ} \mathrm{C}$ for $15 \mathrm{~s}$ and $60{ }^{\circ} \mathrm{C}$ for $1 \mathrm{~min}$ in a StepOne ${ }^{\mathrm{TM}}$ Real-Time PCR System (Applied Biosystems, USA). Real-Time PCR software v.2.2.2 was used for allelic discrimination.

\section{High Resolution Melting (HRM)}

HRM analysis was performed using a StepOne ${ }^{\mathrm{TM}}$ Real-Time PCR System according to the manufacturer's recommendations. Reactions were carried out in a final volume of $10 \mu \mathrm{L}$ containing $20 \eta \mathrm{g}$ of genomic DNA, 0.3 $\mu \mathrm{M}$ of each primer and $1 \mathrm{X}$ MeltDoctor ${ }^{\mathrm{TM}}$ Master Mix (Applied Biosystems, USA). Primers used for exon 10 amplification were published previously (Bastien et al., 2008). Cycling conditions were as follows: initial denaturation at $95^{\circ} \mathrm{C}$ for $10 \mathrm{~min}, 40$ cycles at $95^{\circ} \mathrm{C}$ for $15 \mathrm{~s}, 57^{\circ} \mathrm{C}$ for 30 $\mathrm{s}$ and $60^{\circ} \mathrm{C}$ for $30 \mathrm{~s}$. After denaturation of the PCR products at $95{ }^{\circ} \mathrm{C}$ for $10 \mathrm{~s}$, HRM melting curve data were obtained by continuous fluorescence acquisition from 60 to $95^{\circ} \mathrm{C}$ with at a ramp rate of $0.3 \%$. Melting curves were analyzed with the High Resolution Melt Software v3.0.1 (Applied Biosystems, USA). Since HRM is a mutation screening method, whenever an abnormal melting curve was identified, Sanger sequencing was performed to identify the specific sequence alteration.

\section{Quality Control}

Wild type and mutant p.R337H DNA samples, identified from previous research studies and genotyped by Sanger sequencing in two independent blood samples, were included in each run for assurance and quality control purposes (Table 1). All PCR-RFLP, TaqMan-PCR and HRM analyses were performed in duplicates and Sanger sequencing was bidirectional. All analyses were blinded with respect to the status of previous genotyping results. Three investigators reviewed all genotyping results independently.

\section{Cost analysis}

We used the system of absorption cost analysis based on the technical protocols. Tables were set out in Excel software considering consumables, costs with laboratory personnel, direct and indirect costs associated with the laboratory infrastructure, and losses defined a priori at $10 \%$ (Mahony et al., 2009). The costs of consumables were calculated including reagents and supplies, according to updated prices, in local currency (Gonçalves et al., 2009). Personnel-related costs included estimates of labor hours and salary-related taxes in Brazil. Indirect costs were estimated through the Management Information System (Business Intelligence) of the institution. They included indirect labor costs (employee benefits including occupational medical care), air conditioning, cleaning, building maintenance, security, elevator and electrical power (FerreiraDa-Silva et al., 2012). Costs were calculated considering $100 \%$ use of the installed capacity per run for each of the genotyping techniques performed, according to the equipment available (Table 1). We have not considered costs of acquisition of the equipment, assuming that the infrastructure needed for all of the genotyping methods is already available in a given laboratory. Also, it is important to note that "maximum capacity" was considered for a given type (model) of equipment and may change with different equipment models (i.e. for HRM we have considered maximum capacity of use in a thermal cycler of 48 wells;

Table 1 - Features of each TP53-p.R337H genotyping method.

\begin{tabular}{lcccc}
\hline & Sanger Sequencing $^{\mathrm{a}}$ & PCR-RFLP $^{\mathrm{b}}$ & TaqMan-PCR $^{\mathrm{c}}$ & HRM $^{\mathrm{c}}$ \\
\hline Negative controls (GG genotype) & 0 & 1 & 2 & 3 \\
Positive controls (GA or AA genotypes) $^{\text {Patient samples tested per run }}{ }^{\mathrm{d}}$ & 0 & 2 & 22 & 2 \\
Throughput (full capacity) $_{\text {Total turnaround time (hours) }}^{\mathrm{e}}$ & 47 & 27 & 48 & 21 \\
Hands-on time (hours) & 96 & 62 & 3 & 48 \\
\hline
\end{tabular}

\footnotetext{
${ }^{a} 96-$-well plates

b 2X 31-well gels

${ }^{c} 48$-well plates.

${ }^{\mathrm{d}}$ bidirectional sequencing, duplicate analyses for all other methods

${ }^{\mathrm{e}}$ does not include DNA isolation and quantification.
} 
estimates may differ if a 96-well equipment is used). For the cost analyses performed here we have used the equipment available in our center. The total cost of TP53p.R337H analysis by each method includes all steps necessary to obtain results, including DNA isolation, quantification, all steps of each genotyping method and professional labor cost for sample handling and result interpretation.

Considering that HRM is a mutation screening method and direct DNA sequencing must be performed to confirm the sequence alteration if the melting profile of a given sample differs from that of WT controls, we calculated the HRM costs to analyze 100 patients, in different scenarios of mutation prevalence. For each possible mutation frequency ( 0 to $100 \%$ ), in addition to HRM, we considered costs of Sanger sequencing of the minimal estimated proportion of samples that would have an abnormal melting profile, according to the mutation prevalence in each scenario. A single DNA isolation and quantification step was considered for each individual analyzed, as summarized in Figure 1.

\section{Turnaround and hands-on time analysis}

To establish turnaround times, we considered the total time to perform all steps of each genotyping method using $100 \%$ of the installed capacity per run, including handling, reactions, incubations, centrifugations and interpretation of results. The hands-on time was a fraction of turnaround, comprising the hands-on steps in which an employee needs to be fully dedicated to the activity, i.e. sample handling and direct interpretation of the results.

\section{Results}

To verify the performance of PCR-RFLP, TaqManPCR and HRM for p.R337H genotyping, genomic DNA isolated from 95 peripheral blood samples was processed by all three methods and results compared to those obtained by Sanger sequencing. Among the 95 samples analyzed, 64

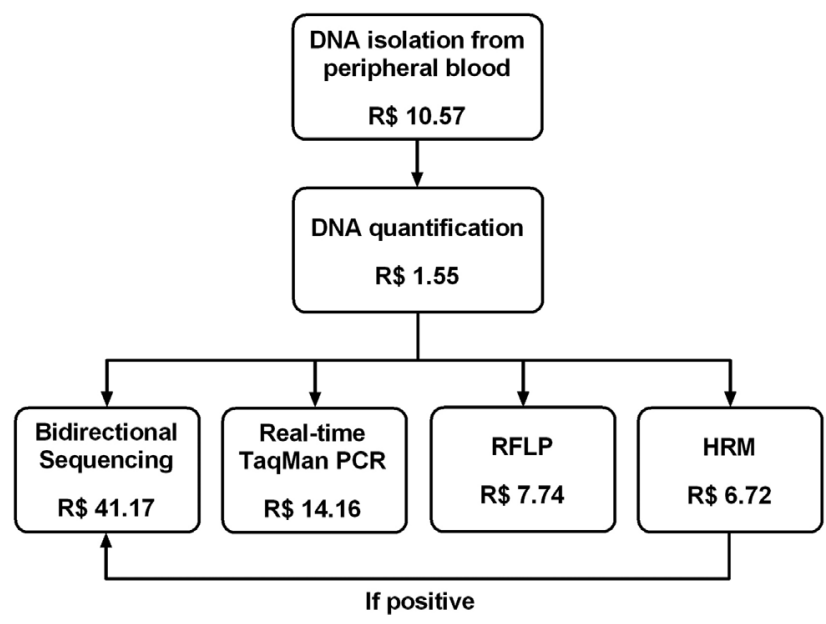

Figure 1 - Costs of each analytical step of TP53-p.R337H genotyping by different methods for one patient. non-carriers (GG genotype, wild-type homozygotes), 30 p.R337H heterozygotes (GA genotype) and 1 p.R337H homozygote (AA genotype) were identified. Results were $100 \%$ concordant with sequencing using all three methods. Representative images of results obtained with the different methodologies are shown in Figure S1.

Cost calculations for each technique were done by an administrator (RPS) and are depicted in Table 2 and Figure 1. Direct sequencing was the most expensive method followed by TaqMan-PCR, PCR-RFLP and HRM. HRM was the least expensive technology, with a cost of $R \$ 18.84$ per patient tested, 2.83 fold less than DNA sequencing. However, it is important to note that this cost does not include the confirmatory sequencing step, needed when an abnormal melting curve is identified. The throughput of each platform used, number of controls included in each run, as well as turnaround and hands-on times needed for genotyping with all methods are summarized in Table 1.

We also assessed costs of all four genotyping strategies taking into account different scenarios of mutation prevalence. Since HRM analysis requires a sequencing step when samples show abnormal melting profiles, there is an increment of overall cost of HRM as the prevalence of positive samples increases. The other three techniques have the same overall cost for a given sample set, independent of mutation prevalence. The costs of genotyping with HRM followed by sequencing (when needed) were lower than all other methods only when the expected mutation prevalence was less than $2.5 \%$, were equal to TaqMan-PCR when mutation frequency in a given sample set was nearly $18 \%$, and equal to sequencing only when the expected mutation frequency was at $83.5 \%$ (Figure 2 and Table S1).

\section{Discussion}

The mutant p.R337H founder allele has been found at a high frequency in patients diagnosed with tumors of the LFS/LFL spectrum (such as adrenocortical, choroid plexus and breast carcinomas) and also in the general population of Southern and Southeastern Brazil (Malkin et al., 1990; Ribeiro et al., 2001; Lalloo et al., 2006; Achatz et al., 2007; Palmero et al., 2008; Garritano et al., 2010; Seidinger et al., 2011; Giacomazzi et al., 2013; Cury et al., 2014; Giacomazzi et al., 2014). This scenario, of a highly prevalent germline mutation in a specific geographic region, is not unusual and has been described for many genetic disorders worldwide (Ewald et al., 2011; Antczak et al., 2013; Pinheiro et al., 2013). In these situations, the use of robust (reliable) and at the same time affordable mutation detection techniques is essential. In the present study, we compared the performance characteristics and costs of four distinct genotyping techniques commonly used to detect the p.R337H founder mutation. Each method has its particular advantages and disadvantages, but genotyping results obtained with all four techniques were fully concordant, demonstrating that all of them can be reliably used for $\mathrm{p} . \mathrm{R} 337 \mathrm{H}$ 
Table 2 - Costs of the TP53-p.R337H analysis for one patient.

\begin{tabular}{lcccc}
\hline Description & HRM $^{\mathrm{c}}$ & PCR-RFLP & TaqMan-PCR & DNA Sequencing \\
\hline Total cost $(\mathrm{R} \$)^{\mathrm{a}}$ & 18.84 & 19.86 & 26.28 & 53.29 \\
Fold increase of cost $^{\mathrm{b}}$ & 1.00 & 1.05 & 1.39 & 2.83 \\
\hline
\end{tabular}

a includes DNA isolation, quantification, all steps of each method and professional labor cost for handling and result interpretation

${ }^{\mathrm{b}}$ in relation to the least expensive method

${ }^{\mathrm{c}} \mathrm{HRM}$ is a screening method and not a direct genotyping method as PCR-RFLP, TaqMan and Sanger sequencing (see Figure 2 for further details on additional cost).

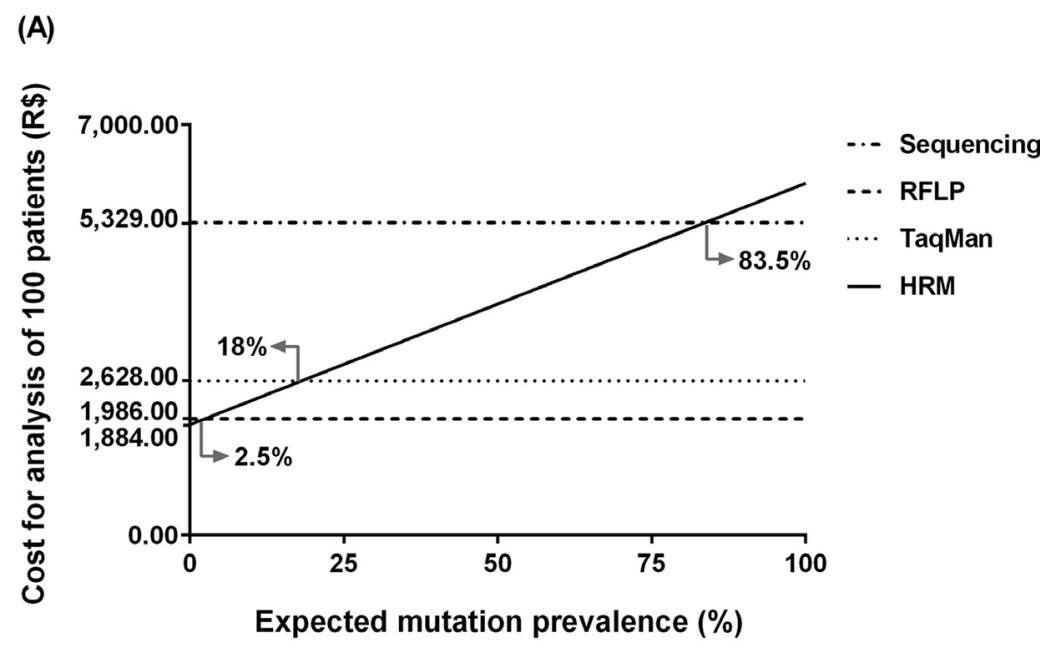

(B)

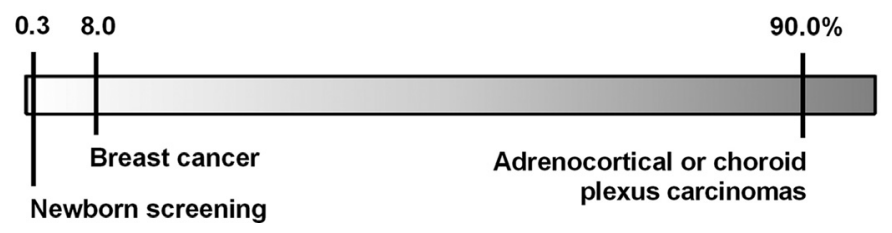

Figure 2 - Cost variation of HRM analysis for screening of the TP53-p.R337H mutation according to estimated mutation prevalence. (A) Cost comparison of Sanger sequencing, TaqMan-PCR and PCR-RFLP. (B) Expected mutation prevalence scenario.

detection. However, our results demonstrate that cost, turnaround and hands-on times can vary significantly with different methods, and a careful analysis should be done in determining which genotyping method is most adequate, depending on the infrastructure available, application and clinical scenario. It is important to emphasize that, as mentioned previously, we have not considered costs of acquisition of the equipments. We considered that there is significant diversity among institutions that could have influenced these costs (i.e. some institutions benefit from tax exemption incentives and others do not) and also recognize that Brazilian laboratories often use core facilities for diagnosis in their institutions, which eliminates the necessity of equipment acquisition.

In our analysis and with our laboratory setup, Sanger sequencing had the highest throughput when compared to other methods, but had the longest turnaround time and the highest cost (for single patient analysis it was 2.83 times higher compared to the least expensive procedure). Sanger sequencing requires advanced instrumentation, but the entire process is semi-automated, and both the laboratory protocols and result interpretation require significant hands-on dedication time. Despite these limitations, Sanger sequencing by capillary electrophoresis is still considered the gold standard in single gene mutation analysis in many centers and has been used in clinical genetic testing for many years. It is a robust, highly reproductive approach ideal for identification of mutations in a given DNA sequence, without necessity of previous interrogation of a specific mutation.

HRM, on the other hand is a mutation screening method, also widely used in clinical diagnostics, but it requires confirmation of genotype with a second method whenever a melting abnormality is identified. Several studies validated HRM for analysis of germline TP53 mutations using different sample types and always demonstrating high sensitivity (81-100\%) and specificity (83-99\%) (Kry- 
puy et al., 2007; Bastien et al., 2008; Garritano et al., 2009). In the present study, sensitivity and sensibility of HRM analysis reached $100 \%$, probably due to the use of high quality DNA obtained from leukocytes and to the short amplicon length used $(87 \mathrm{bp})$. Compared to the other techniques assessed here, HRM had the lowest cost per patient, with a turnaround of nearly 5 hours, offering a convenient closed-tube method to assess the presence of single-base sequence variations. However, good laboratory practice recommends that amplicons with altered melting profiles be sequenced to identify which specific mutation or polymorphism is present, since different heterozygotes may produce similar melting curves. Thus, HRM is clearly suitable for mutation screening in populations with lower mutation prevalence (in the case of analyses directed to one single mutation) or with less disease-associated variants (in the case of mutation screening of an entire gene) (Li et al., 2011).

In this study, we demonstrate that HRM analysis is cheaper than any of the other methods used when the predicted mutation prevalence in a given sample set is less than $2.5 \%$, and less expensive than TaqMan-PCR or DNA sequencing when the estimated mutation prevalence reaches close to $18 \%$. Thus, in Brazil, where the prevalence of p.R337H has been reported for several different sample sets, HRM would be an excellent strategy for mutation screening in the general population (mutation prevalence reported at $0.3 \%$ in a newborn screening program) and could also be considered in women with breast cancer (mutation prevalence up to $8 \%$, depending on age at cancer diagnosis) along with other methods in this second group (Custodio et al., 2013; Cury et al., 2014; Giacomazzi et al., 2014).

TaqMan-PCR, on the other hand, had the lowest turnaround and hands-on times in our study and has the great advantage of allowing simultaneous amplification and allelic discrimination in about 3 hours, without any further manual steps. However, as HRM, it had a low throughput in our study, due to the 48 wells real-time platform used and the need of performing reactions in duplicates, which both increase the overall time of analysis for large sample sets. TaqMan-PCR has a lower cost than Sanger sequencing and HRM when the expected mutation prevalence in the study population is above $18 \%$ (i.e. in some families with phenotypic criteria for $\mathrm{Li}$-Fraumeni or Li-Fraumeni-like syndrome). For these situations and especially when results are needed quickly it is an excellent diagnostic approach.

Finally, PCR-RFLP showed reasonable costs and has the important advantage of minimal requirements in terms of investment in instrumentation. In addition, genotyping can be easily done by visualization of restriction fragments by gel electrophoresis, for which no specific software is needed. The most important disadvantage of PCR-RFLP, perhaps, is that it is a relatively time-consuming method, consisting of several sequential, and mostly not automated steps. In general, however, it is considered a simple, inexpensive and accurate method for genotyping, useful in small research studies and for laboratories that do not have advanced infrastructure or have limited financial resources.

In this study we compared performance, cost and turnaround time of Sanger sequencing, PCR-RFLP, TaqMan-PCR and HRM in the detection of a cancer predisposing founder mutation, TP53-p.R337H. This strategy, and results obtained here can be applied to other sequence variants associated with genetic disorders in high risk populations.

We conclude that multiple methodologies are suitable for the detection of TP53-p.R337H and genotyping results obtained in this study with these different strategies where fully concordant. The method of choice to be used in a given scenario will depend on the available laboratory infrastructure, acceptable time for result reporting and especially estimated mutation prevalence in the sample set to be analyzed.

\section{Acknowledgments}

MFK was supported by a fellowship from CNPq (Brazil). The study was supported in part by grants from GlaxoSmithKline Oncology (Ethnic Research Initiative Grant Award 2009), U.K.; CNPq, Brazil (grant 307779 2009-2); FAPERGS-PPSUS (grants 09/0103-0 and 002/2013), FAPERGS PRONEX (grant 10/0051-9), and Fundo de Incentivo a Pesquisa e Eventos, Hospital de Clínicas de Porto Alegre (GPPG 08080), Brazil.

\section{References}

Achatz MI, Olivier M, Le Calvez F, Martel-Planche G, Lopes A, Rossi BM, Ashton-Prolla P, Giugliani R, Palmero EI, Vargas FR, et al. (2007) The TP53 mutation, R337H, is associated with Li-Fraumeni and Li-Fraumeni-like syndromes in Brazilian families. Cancer Lett 245:96-102.

Antczak A, Kluzniak W, Wokolorczyk D, Kashyap A, Jakubowska A, Gronwald J, Huzarski T, Byrski T, Deogonekbniak T, Masojc B, et al. (2013) A common nonsense mutation of the BLM gene and prostate cancer risk and survival. Gene 532:173-176.

Bastien R, Lewis TB, Hawkes JE, Quackenbush JF, Robbins TC, Palazzo J, Perou CM and Bernard PS (2008) Highthroughput amplicon scanning of the TP53 gene in breast cancer using high-resolution fluorescent melting curve analyses and automatic mutation calling. Hum Mutat 29:757764.

Cury NM, Ferraz VE and Silva WA (2014) TP53 p.R337H prevalence in a series of Brazilian hereditary breast cancer families. Hered Cancer Clin Pract 12:8.

Custodio G, Parise GA, Kiesel Filho N, Komechen H, Sabbaga CC, Rosati R, Grisa L, Parise IZ, Pianovski MA, Fiori CM, et al. (2013) Impact of neonatal screening and surveillance for the TP53 R337H mutation on early detection of childhood adrenocortical tumors. J Clin Oncol 31:2619-2626. 
Euhus DM and Robinson L (2013) Genetic predisposition syndromes and their management. Surg Clin North Am 93:341-362.

Ewald IP, Izetti P, Vargas FR, Moreira MA, Moreira AS, Moreira-Filho CA, Cunha DR, Hamaguchi S, Camey SA, Schmidt A, et al. (2011) Prevalence of the BRCA1 founder mutation c.5266dupin Brazilian individuals at-risk for the hereditary breast and ovarian cancer syndrome. Hered Cancer Clin Pract 9:12.

Ferreira-Da-Silva AL, Ribeiro RA, Santos VCC, Elias FTS, d'Oliveira ALP and Polanczyk CA (2012) Diretriz para análises de impacto orçamentário de tecnologias em saúde no Brasil. Cad Saude Publica 28:1223-1238.

Garritano S, Gemignani F, Palmero EI, Olivier M, Martel-Planche G, Le Calvez-Kelm F, Brugieres L, Vargas FR, Brentani RR, Ashton-Prolla P, et al. (2010) Detailed haplotype analysis at the TP53 locus in p.R337H mutation carriers in the population of Southern Brazil: Evidence for a founder effect. Hum Mutat 31:143-150.

Garritano S, Gemignani F, Voegele C, Nguyen-Dumont T, Le Calvez-Kelm F, De Silva D, Lesueur F, Landi S and Tavtigian SV (2009) Determining the effectiveness of high resolution melting analysis for SNP genotyping and mutation scanning at the TP53 locus. BMC Genet 10:e5.

Giacomazzi J, Graudenz MS, Osorio CABT, Koehler-Santos P, Palmero EI, Zagonel-Oliveira M, Michelli RAD, Neto CS, Fernandes GC, Achatz MIWS, et al. (2014) Prevalence of the TP53 p.R337H mutation in breast cancer patients in Brazil. PLoS One 9:e99893.

Giacomazzi J, Koehler-Santos P, Palmero EI, Graudenz MS, Rivero LF, Lima E, Pütten ACK, Hainaut P, Camey SA, Michelli RD, et al. (2013) A TP53 founder mutation, p.R337H, is associated with phyllodes breast tumors in Brazil. Virchows Arch 463:17-22.

Gonçalves MA, Zac JI and de Amorim CA (2009) Hospital strategic management: The use of costing process in Health. Rev Adm FACES J 8:161-179.

Gonzalez KD, Buzin CH, Noltner KA, Gu D, Li W, Malkin D and Sommer SS (2009) High frequency of de novo mutations in Li-Fraumeni syndrome. J Med Genet 46:689-693.

Krypuy M, Ahmed AA, Etemadmoghadam D, Hyland SJ, DeFazio A, Fox SB, Brenton JD, Bowtell DD, Dobrovic A and Group AOCS (2007) High resolution melting for mutation scanning of TP53 exons 5-8. BMC Cancer 7:e168.

Lalloo F, Varley J, Moran A, Ellis D, O'dair L, Pharoah P, Antoniou A, Hartley R, Shenton A, Seal S, et al. (2006) BRCA1, BRCA2 and TP53 mutations in very early-onset breast cancer with associated risks to relatives. Eur J Cancer 42:1143-1150.

Li BS, Wang XY, Ma FL, Jiang B, Song XX and Xu AG (2011) Is High Resolution Melting Analysis (HRMA) accurate for detection of human disease-associated mutations? A meta analysis. PLoS One 6:e28078.

Livak KJ (1999) Allelic discrimination using fluorogenic probes and the 5' nuclease assay. Genet Anal 14:143-149.

Mahony JB, Blackhouse G, Babwah J, Smieja M, Buracond S, Chong S, Ciccotelli W, O'Shea T, Alnakhli D, GriffithsTurner M, et al. (2009) Cost analysis of multiplex PCR testing for diagnosing respiratory virus infections. J Clin Microbiol 47:2812-2817.
Malkin D, Li FP, Strong LC, Fraumeni Jr. JF, Nelson CE, Kim DH, Kassel J, Gryka MA, Bischoff FZ, Tainsky MA, et al. (1990) Germ line p53 mutations in a familial syndrome of breast cancer, sarcomas, and other neoplasms. Science 250:1233-1238.

Narayanan S (1991) Applications of restriction fragment length polymorphism. Ann Clin Lab Sci 21:291-296.

Palmero EI, Schüler-Faccini L, Caleffi M, Achatz MI, Olivier M, Martel-Planche G, Marcel V, Aguiar E, Giacomazzi J, Ewald IP, et al. (2008) Detection of R337H, a germline TP53 mutation predisposing to multiple cancers, in asymptomatic women participating in a breast cancer screening program in Southern Brazil. Cancer Lett 261:21-25.

Petitjean A, Mathe E, Kato S, Ishioka C, Tavtigian SV, Hainaut P and Olivier M (2007) Impact of mutant p53 functional properties on TP53 mutation patterns and tumor phenotype: Lessons from recent developments in the IARC TP53 database. Hum Mutat 28:622-629.

Pinheiro M, Pinto C, Peixoto A, Veiga I, Mesquita B, Henrique R, Lopes P, Sousa O, Fragoso M, Dias L, et al. (2013) The MSH2 c.388_389del mutation shows a founder effect in Portuguese Lynch syndrome families. Clin Genet 84:244250.

Reed GH, Kent JO and Wittwer CT (2007) High-resolution DNA melting analysis for simple and efficient molecular diagnostics. Pharmacogenomics 8:597-608.

Ribeiro RC, Sandrini F, Figueiredo B, Zambetti GP, Michalkiewicz E, Lafferty AR, DeLacerda L, Rabin M, Cadwell C, Sampaio G, et al. (2001) An inherited p53 mutation that contributes in a tissue-specific manner to pediatric adrenal cortical carcinoma. Proc Natl Acad Sci U S A 98:9330-9335.

Seidinger AL, Mastellaro MJ, Paschoal Fortes F, Godoy Assumpção J, Aparecida Cardinalli I, Aparecida Ganazza M, Correa Ribeiro R, Brandalise SR, Dos Santos Aguiar S and Yunes JA (2011) Association of the highly prevalent TP53 R337H mutation with pediatric choroid plexus carcinoma and osteosarcoma in Southeast Brazil. Cancer 117:2228-2235.

Wittwer CT, Reed GH, Gundry CN, Vandersteen JG and Pryor RJ (2003) High-resolution genotyping by amplicon melting analysis using LCGreen. Clin Chem 49:853-860.

\section{Internet Resources}

IARC TP53 Database, http://p53.iarc.fr/Download/TP53_DirectSequencing_IARC.pdf (March 23, 2015)

\section{Supplementary Material}

The following online material is available for this article:

Table S1 - Costs of TP53-p.R337H genotyping for 100 patients

Figure S1 - Results obtained with the different genotyping methodologies

This material is available as part of the online article from http://www.scielo.br/gmb

Associate Editor: Pierre Hainaut

License information: This is an open-access article distributed under the terms of the Creative Commons Attribution License (type CC-BY), which permits unrestricted use, distribution and reproduction in any medium, provided the original article is properly cited. 